\title{
Quantum Measurement Cannot Be a Local Physical Process
}

\author{
Hitoshi Inamori \\ Société Générale, Puteaux, France \\ Email: hitoshi.inamori@gmail.com
}

How to cite this paper: Inamori, H. (2019) Quantum Measurement Cannot be a Local Physical Process. Journal of Quantum Information Science, 9, 171-178. https://doi.org/10.4236/jqis.2019.94009

Received: October 24, 2019

Accepted: December 10, 2019

Published: December 13, 2019

Copyright (c) 2019 by author(s) and Scientific Research Publishing Inc. This work is licensed under the Creative Commons Attribution International License (CC BY 4.0).

http://creativecommons.org/licenses/by/4.0/

\begin{abstract}
According to quantum mechanics, the outcome of an experiment exists relative to an Experimenter who performs a measurement on the system under study. Witnessing the outcome of an experience requires the measurement on a physical system whose size must match the complexity of the Experimenter's observation. We argue that such a physical system must have a certain space-time extension so that it can encode the rich and complex data embedded in the witnessed experience. The complementarity principle in quantum mechanics leads us to conjecture that the observable events constituting an experience have space-like separation with each other. This seems to be in contradiction with our perceived locality of physical laws, and encourages us to think that the act of measurement is not a physical process, in the sense that a measurement outcome witnessed by an Experimenter is not necessarily related to the physical description of the Experimenter observed from the outside.
\end{abstract}

\section{Keywords}

Quantum Measurement Theory, Locality of Quantum Mechanical Laws

\section{Introduction}

According to quantum mechanics [1] [2], the evolution of the state of a physical system obeys two laws: a linear and continuous evolution with respect to time while the physical system is not being observed, and a discontinuous and non-linear probabilistic map when a measurement is performed on the system, i.e. when the system is being observed.

The theory remains however vague about who or what is entitled to perform such a measurement [3]. In general this does not cause an issue as we assume that any actor who can perform a measurement is a classical system, i.e. a system so large that once it has observed a given outcome, this fact becomes irreversible 
for the whole universe [4] [5]. In other words, the result observed by an actor becomes a universal truth for any other observer. With such an assumption, all observers are equivalent and there is indeed no need to precise who is performing a given measurement on which system.

In most practical cases this approximation does not cause an issue. However any observer, regardless of its size, remains a finite physical system and its evolution, seen from the outside by another observer, should be continuous and linear. This is in contradiction with the discontinuous evolution experienced by the inner observer, and this paradox has been captured in particular in the Wigner's friend paradox [6] and more recently in an extended version of it [7].

This paradox tells us that in quantum theory it is fundamentally important to specify precisely who is the observer and which physical system is being observed [6]. Surprisingly, at least in the author's knowledge, there is little discussion in the literature about what constitutes exactly the physical system being observed by a given observer. This could be explained by the above implied assumption that all observers are equivalent in the mainstream acceptation of quantum mechanics.

The goal of the present paper is to examine what constitutes the physical system being observed by the Experimenter and to discuss its characteristics. We assert that the outcome witnessed by an Experimenter is in general sufficiently complex so that it requires a set of observable events with a certain extension in space-time. In order to access these information, the observer therefore needs to perform a measurement on observables that are separated in space-time with each other. Quantum measurement theory does not specify how such a joint measurement is done, which observables are measured, or whether there is a constraint on the space-time extension of the system being measured. In particular, can we say something about the choice of the observables that are part of the measured system, and the nature of the space-time separation between these observables?

We find that quantum mechanics complementarity principle [8] strongly suggests that these observables must have space-like separation. However, quantum mechanics does not tell whether there is a limitation on the distance between these observables, as long as they are space-like separated. This result is at odds with our intuition. How can a unified experience result from measurement of systems which are space-like separated?

It is also in contradiction with the locality principle, which states that one cannot convey information at a speed faster than light. Indeed, if I could observe the present state of a physical system located very far away, I could act on that information and violate the locality principle.

We propose few alternatives that could solve this contradiction, but we also argue that there might not be such a contradiction to start with. Even if an Experimenter has a subjective experience of what is happening at light-years distance now, as long as his actions seen from the outside does not betray this experience, we argue that physical locality is not violated. And seen from the 
outside, the behaviour of the Experimenter can be fully explained by physical laws without involving the subjective experience witnessed by the Experimenter.

These arguments lead us to accept that the observation made by an Experimenter is a subjective concept, enjoying a certain independence from the physical support containing the information, and the physical reaction of the Experimenter following the observation.

\section{An Observation Cannot Be Limited to a Measurement at a Single Point in Space Time}

Whenever we observe the outcome of an experiment, our experience is never limited to the single experimental quantities we were proposing to measure. Instead, our experience encodes all the contexts accompanying the experiment, such as the experimental setup, the preparation of the initial state, the state of the environment, etc. All these information constitute a unified experience which cannot be decomposed into smaller independent experiences. Because of its complexity, the information embedded in this experience cannot be encoded into a single point in space-time but requires a physical system with a certain extension in space-time to encode it.

Let's take an example. Suppose that an Experimenter wants to determine whether a photon impinges a given photo-detection unit. The outcome of such experiment would usually have a simple structure as a "true" or "false" statement. However, this description is an oversimplification corresponding to a restricted view of what the Experimenter actually witnesses as we are completely ignoring the output of any other measurement devices that could be present, or the eventuality that the photo-detector explodes for instance (in which case does this count as "true" or "false"?).

Finally the information "true" or "false" by itself is meaningless without context. It is only given the context of the experimental setup, for instance the placement of the different experimental instruments and the preparation of the initial state, that the outcome "true" or "false" has a meaning. As such, the context of the experiment should be part of the experiment outcome [9]. The context is usually omitted from the experimental result as it is considered to be an unmovable assumption, but this is a questionable assumption: in theory, the context of the experiment is encoded in a physical medium, and it can itself evolve as the experiment unfolds [10].

The purpose of this paper is not to discuss how the output of an experiment should be encoded and why. What we want to stress on here is that the information returned by observing the outcome of an experiment has a complex structure, which cannot be encoded in a single elementary physical system:

Conjecture 1. Describing the outcome of an experiment requires a data structure which encodes everything witnessed by the Experimenter. This entails a data structure which cannot be encoded in an elementary physical system such as an elementary particle.

It seems therefore very unlikely that the full outcome of an experiment can 
be encoded into a physical system that is located at a single point in space-time. Albeit not proven, it is far more intuitive to assume that the encoding of the outcome of an experiment requires a physical system that has a non-zero extension in space-time:

Definition 1. We call an observable event a physical observable quantity at a given space time coordinate $(t, x)$ in a given reference frame.

Conjecture 2. Observing the outcome of an experiment requires the measurement of a physical system that has a non-zero extension in space-time, such as a set of elementary observable events which have a non-zero space-time separation between themselves.

For instance, we could imagine that the information obtained by the Experimenter could be encoded in the spin of a series of electrons $i=1,2, \cdots$ located at some locations in space-time $\left\{\left(t_{1}, x_{1}\right),\left(t_{2}, x_{2}\right), \cdots\right\}$, where the $\left(t_{i}, x_{i}\right)$ are distinct, although such representation is unlikely to be the actual one.

\section{An Observation Is Equivalent to a Measurement of Observable Events that Have Space-Like Separation}

Observation of the outcome of a real experiment implies a measurement of observable events that are separated from each other. Can we say more about the nature of this separation?

At first we are tempted to think that these observable events are "linked" with each other by physical mean, for instance by the exchange of elementary particles, so that the set of observable events presents somehow a "coherent and unified" view of the experiment's outcome. This would imply in particular that these observable events are time-like separated with each other (we recall that events $\left(t_{A}, x_{A}\right)$ and $\left(t_{B}, x_{B}\right)$ have time-like separation if $\left\|x_{B}-x_{A}\right\|^{2} \leq c^{2}\left(t_{B}-t_{A}\right)^{2}$ where $c$ is speed of light, otherwise they have space-like separation).

However, the properties of quantum measurement, and in particular, the complementary principle, imply on the contrary that these observable events should be separated so that no physical interaction is possible between them.

To see this, suppose that we have an experimental outcome whose observation requires the measurement of two observable systems $A$ and $B$. Suppose that we want to observe the state of these systems at space-time events $\left(t_{A}, x_{A}\right)$ and $\left(t_{B}, x_{B}\right)$, respectively, and that physical interaction between $A$ and $B$ is allowed between $\left(t_{A}, x_{A}\right)$ and $\left(t_{B}, x_{B}\right)$.

Now suppose that one can learn the state of $A$ at location $\left(t_{A}, x_{A}\right)$ and the state of $B$ location $\left(t_{B}, x_{B}\right)$ without affecting the states of $A$ and $B$ (Figure 1 ).

This would clearly violate the complementary principle of quantum mechanics. To see this, suppose that the systems $A$ and $B$ are two qubits, and that $A$ is prepared in the state $|\psi\rangle$ which is chosen randomly between the states of an orthonormal basis $\{|0\rangle,|1\rangle\}$ or the states of the conjugate basis $\left\{|+\rangle=\frac{1}{\sqrt{2}}(|0\rangle+|1\rangle),|-\rangle=\frac{1}{\sqrt{2}}(|0\rangle-|1\rangle)\right\}$.

The complementarity principle tells that no measurement is possible that allows 


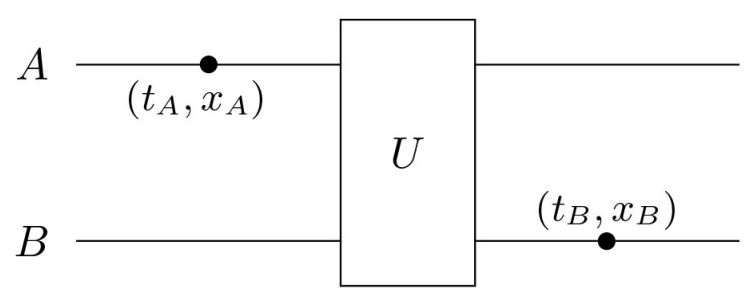

Figure 1. Example with two observable events.

the discrimination between the state $|0\rangle$ or $|1\rangle$ or between the state $|+\rangle$ or $|-\rangle$ if one does not know beforehand from which set $\{|0\rangle,|1\rangle\}$ or $\{|+\rangle,|-\rangle\}$ the initial state for $A$ has been chosen.

Now, if we could know the state of the first qubit at space-time location $\left(t_{A}, x_{A}\right)$ and the state of the second qubit at space-time location $\left(t_{B}, x_{B}\right)$ and if we allowed an interaction to take place between the two qubits between the two observations, then we could swap the two qubits and apply the Hadamard gate on the second qubit. Doing so one could measure perfectly the initial state $|\psi\rangle$ in the basis $\{|0\rangle,|1\rangle\}$ and then in the conjugate basis $\{|+\rangle,|-\rangle\}$, which is in clear contradiction with the complementarity principle (Figure 2). The first qubit cannot be known at spacetime location $\left(t_{A}, x_{A}\right)$ and the second qubit known at time $\left(t_{B}, x_{B}\right)$ while allowing interaction between the two qubits between the two events.

Property 1. A measurement on a physical system composed of elementary observables at different space-time events is possible only if physical interaction between the elementary observable systems is not allowed in the time interval between these events.

Remark: Of course, there is a correct and conventional quantum mechanical description corresponding to a measurement of $A$ at $\left(t_{A}, x_{A}\right)$ and $B$ at $\left(t_{B}, x_{B}\right)$. In such a description, we would introduce two ancillary quantum systems $A$ ' and $B$ which come into interaction with the systems $A$ and $B$ at space-time location $\left(t_{A}, x_{A}\right)$ and $\left(t_{B}, x_{B}\right)$. By construction, we assume that there is no further interaction possible for the ancillary systems $A^{\prime}$ and $B^{\prime}$ which are measured at some subsequent arbitrary time $t$. In particular, $A^{\prime}$ and $B$ do no interact with each other at all after their respective interaction with $A$ and $B$ in this conventional representation. As such, although we do not set any restriction on the interaction between the systems $A$ and $B$ between the events $\left(t_{A}, x_{A}\right)$ and $\left(t_{B}, x_{B}\right)$, this conventional quantum mechanical representation is in line with Property 1 , if we note that the actually observed systems are not $A$ and $B$ but the ancillary systems $A^{\prime}$ and $B$ : a measurement on a physical system composed of elementary observable events $A^{\prime}$ at $\left(t_{A}, x_{A}\right)$ and $B^{\prime}$ at $\left(t_{B}, x_{B}\right)$ is possible as $A^{\prime}$ and $B^{\prime}$ do not physically interact with each other between these events (Figure 3 ).

We have argued in this section that an observation of an experimental outcome involves the measurement of observable events which are located so that no physical interaction is possible between these events. The simplest and surest way to ensure that such interaction is impossible, is to have these events 


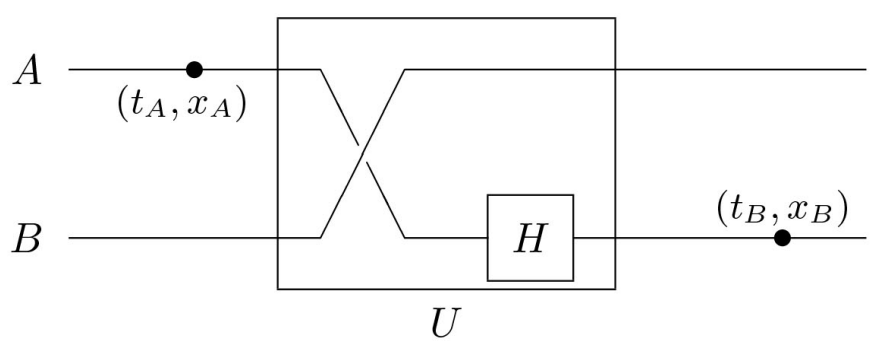

Figure 2. Intraction cannot be allowed between two observable events.

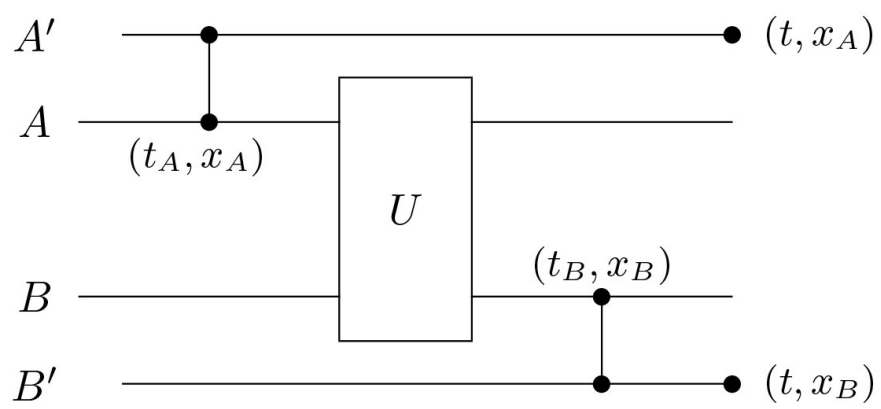

Figure 3. Correct description of measurement of two observable events.

being separated space-like, as this would ensure that no physical medium can link the observed events. We therefore propose that:

Conjecture 3. The observation of an experiment outcome is equivalent to a measurement of space-like separated observable events.

\section{Is Measurement a Local Physical Process?}

The observation witnessed by an Experimenter is the outcome of measurement made on observable events that are space-like separated. If there is no limitation on the extent of this separation, there is no theoretical reason why an Experimenter could not witness very remote observable events such as an event on Earth and an event on a distant star light-years away, as long as these events have space-like separation. This is of course at odds with our daily experience, and our intuition tells us that such observation would be in contradiction with the locality principle.

To circumvent this apparent contradiction, we could add an ad-hoc limitation on the extent of the separation that is allowed between events that can be observed conjointly. If we are told that an Experimenter can witness observable events that have a space-like separation, but only within an extension of $20 \mathrm{~cm}$, then the contradiction-albeit still existing in theory-would seem much more acceptable.

Another similar alternative would be to postulate that the act of measurement takes some finite time duration to complete. The observation does still reflect observable events that have space-like separation, but we suppose that there is an incompressible time duration required to integrate these data into the observation witnessed by the Experimenter. If we assume that this time duration is larger than the time required to establish physical interaction between the observed 
systems, then the locality issue would be resolved: by the time the Experimenter acts based on witnessed observation, enough time would have been spent so that the space-like separated observable events have moved into the time-like past from the Experimenter's subsequent action.

These ideas based on a limitation to circumvent locality issues are appealing. However, Quantum Mechanics does not offer any mechanism or laws that could lead to these limitations. Is there a time duration required to perform a measurement (here we are not referring to the time needed for the measuring apparatus to interact with the physical system under study, but the time required to observe the ancillary quantum system in the final step of the observation)? If so what is this time and is it dependent on the space-like extension of the physical system one is measuring? Is there a mechanism that requires that there is a repeated physical interaction between observables that are part of the unified experience witnessed by the Experimenter? In this case, how can we distinguish the interactions which contribute to the unified experience and the ones which do not? Also, the physical system which was encoding the witnessed information may have evolved by the time the information is actually witnessed by the Experimenter. Isn't it strange that one can be aware of the information that has no physical support encoding it by the time it is witnessed?

Now, more fundamentally, are these limitations on measurement truly required to avoid issues with physical locality? We could argue that physical locality is preserved in practice as long as the Experimenter's actions, seen from the outside, do not depend on events that are space-like separated. And seen from the outside, the actions performed by the Experimenter can be fully explained as a combined result (however complex) of elementary physical processes: indeed, the Experimenter seen from the outside is a physical system like any other. Therefore, by construction, the actions of the Experimenter are consistent with physical locality because it can be explained as a consequence of known physical interactions with its environment. The physical locality is preserved regardless of whether the Experimenter is aware or not of the observable events that are space-like separated: as long as this knowledge does not transpire to another experimenter, we could defend that physical locality is preserved. In a way, the subjective experience of one Experimenter is of no importance to another Experimenter [11].

We can summarize our discussion as follows:

\section{Proposition 1.}

- An Experimenter witnesses observable events that are space-like separated.

- There is no theoretical limitation known on this space-like separation.

- However, seen from the outside, the action of the Experimenter based on his observations does not violate the locality principles.

The Experimenter has a unified experience of what he can observe from an experiment. Our discussion above leads us to think that this unified experience does not necessarily correspond to anything unified if we observe the Experimenter as a physical system from the outside. Rather, the different pieces of information 
are encoded in observable events that are space-like separated. Seen from the outside, the behaviour of the Experimenter can be explained using physical laws, via local interactions on separate physical systems that convey different pieces of information. The outsider does not need to assume the existence of a unified view witnessing space-like separated observable events. As such, we could argue that the outcome of a measurement is purely subjective: the subjective experience may reflect observable events that are space-like separated, but it does not lead to non-locality. The externally visible behaviour of the Experimenter is not the consequence of the subjective experience made by the Experimenter. Seen from the outside, the Experimenter is just another physical mechanism interacting locally with its immediate physical environment.

Quantum measurement, the process leading to this subjective experience, is not a local process as it involves space-like separated events. This non-locality is however not an issue as the description of the observer as a physical system does not depend on this subjective experience. In this sense, we conjecture that quantum measurement-a key feature of quantum physics-is not a local physical process.

\section{Conflicts of Interest}

The author declares no conflicts of interest regarding the publication of this paper.

\section{References}

[1] Heisenberg, W. (1930) The Physical Principles of the Quantum Theory. Dover Publications, New York.

[2] Von Neumann, J. (1955) Mathematical Foundations of Quantum Mechanics. Princeton University Press, Princeton.

[3] Bell, J.S. (1981) Quantum Mechanics for Cosmologists, Quantum Gravity 2. Clarendon Press, Oxford.

[4] Bohr, N. (1928) The Quantum Postulate and the Recent Development of Atomic theory. Nature, 121, 580-590. https://doi.org/10.1038/121580a0

[5] Heisenberg, W. (1958) The Copenhagen Interpretation of Quantum Theory, Physics and Philosophy. Harper \& Row, New York

[6] Wigner, E.P. (1967) Remarks on the Mind-Body Question, in Symmetries and Reflections. Indiana University Press, Bloomington.

[7] Frauchiger, D. and Renner, R. (2018) Quantum Theory Cannot Consistently Describe the Use of Itself. Nature Communications, 9, 3711.

https://doi.org/10.1038/s41467-018-05739-8

[8] Bohr, N. (1934) Atomic Theory and the Description of Nature. Cambridge University Press, Cambridge.

[9] Inamori, H. (2015) Defining the Observed World in Quantum Mechanics. arXiv:1507.01102.

[10] Inamori, H. (2018) The Issue with the Initial State in Quantum Mechanics. arXiv: 1810.11516

[11] Inamori, H. (2018) Quantum Mechanics Allows Undetectable Inconsistencies in Witnessed Events. arXiv:1801.05317. 